\title{
The NSP14/NSP10 RNA repair complex as a Pan-coronavirus therapeutic target
}

\author{
Gergely Rona $\mathbb{D}^{1,2,3,11}$, Andras Zeke ${ }^{1,2,4,11}$, Bearach Miwatani-Minter $\mathbb{D}^{1,2}$, Maren de Vries $\mathbb{D}^{5}$, Ramanjit Kaur ${ }^{5}$, Austin Schinlever (1D ${ }^{5}$, \\ Sheena Faye Garcia $\mathbb{1}^{1,2}$, Hailey V. Goldberg ${ }^{1,2}$, Hui Wang ${ }^{6,7}$, Thomas R. Hinds ${ }^{6,7}$, Fabrice Bailly ${ }^{8}$, Ning Zheng ${ }^{6,7}$, Philippe Cotelle ${ }^{8,9}$, \\ Didier Desmaële ${ }^{10}$, Nathaniel R. Landau ${ }^{5}$, Meike Dittmann $\mathbb{1}^{5 凶}$ and Michele Pagano $\mathbb{D}^{1,2,3 凶}$
}

(c) The Author(s) 2021

The risk of zoonotic coronavirus spillover into the human population, as highlighted by the SARS-CoV-2 pandemic, demands the development of pan-coronavirus antivirals. The efficacy of existing antiviral ribonucleoside/ribonucleotide analogs, such as remdesivir, is decreased by the viral proofreading exonuclease NSP14-NSP10 complex. Here, using a novel assay and in silico modeling and screening, we identified NSP14-NSP10 inhibitors that increase remdesivir's potency. A model compound, sofalcone, both inhibits the exonuclease activity of SARS-CoV-2, SARS-CoV, and MERS-CoV in vitro, and synergistically enhances the antiviral effect of remdesivir, suppressing the replication of SARS-CoV-2 and the related human coronavirus OC43. The validation of top hits from our primary screenings using cellular systems provides proof-of-concept for the NSP14 complex as a therapeutic target.

Cell Death \& Differentiation (2022) 29:285-292; https://doi.org/10.1038/s41418-021-00900-1

\section{INTRODUCTION}

Coronaviruses (CoVs) are positive strand RNA viruses with a replication property unique for RNA viruses - proofreading, executed by a viral exonuclease (ExoN). The proofreading function, which depends on the ExoN activity, allows for maintenance of the large CoV RNA genome by decreasing the mutation rate of the error-prone viral RNA-dependent RNA polymerase. ExoN activity is conferred by the NSP14-NSP10 complex (NSP14/10), in which NSP14, a bifunctional dsRNA exonuclease/guanosine $5^{\prime}$ methyltransferase protein, acts as the catalytic subunit activated by NSP10. The sequence and structure of NSP14 ExoN domain is highly divergent from most cellular exonuclease enzymes, including its closest mammalian relative TREX1, a DNA exonuclease. This disparity makes NSP14/10 an ideal anti-viral drug target. Importantly, the ExoN catalytic site and its surroundings are highly conserved across most CoVs (Supplementary Fig. 1A, B), suggesting the potential for pan-CoV inhibition. The ExoN domain of NSP14 resembles that of a eukaryotic DEDD exonuclease, which, to carry out its nucleotide excision, relies on negatively charged amino acids (in general, three aspartic acids and one glutamic acid) and bivalent metal ions. Specifically, NSP14 has been shown to rely on $\mathrm{Mg}^{2+}$ for its catalytic activity [1-3]. Mutations in NSP14 that impair proofreading function have been shown to critically reduce viral fitness [4-8]. Therefore, inhibition of ExoN is expected to be detrimental for CoV replication. In addition, based on genetic studies ExoN inhibition is expected to increase $\mathrm{CoV}$ sensitivity to chain terminating and mutagenic nucleotides [6, 9-11]. Using a novel, FRET-based exonuclease assay, we have screened small molecule compounds to identify NSP14/10 inhibitors. We then tested the antiviral potency of our best hits using viral replication assays with HCoV-OC43 and SARS-CoV-2. There results of these studies is presented herein.

\section{RESULTS}

The in vitro catalytic activity of NSP14/10 was previously mostly detected in gel-based assays, which are not amenable for highthroughput screenings $[1,3,12]$. We designed a novel, FRET-based exonuclease assay that allows high throughput assessment of potential NSP14/10 inhibitory compounds. We used dsRNA probes with sufficiently low $\mathrm{Tm}$, labeled with a fluorophore and a quencher at a physical proximity (Fig. 1A, Supplementary Fig. 2A, $B$, and Table S1). NSP14/10 recognizes the terminal mismatch and removes bases from the $3^{\prime}$ end of the substrate strand until the two RNA strands separate, and the fluorescence signal increases. This assay provides a more accessible alternative to the recently described mass-spectrometry based screening method [13]. Using purified, recombinant NSP14/10 from either SARS-CoV-2, SARSCoV, or MERS-CoV (Supplementary Fig. 2A), the reaction reached

\footnotetext{
${ }^{1}$ Department of Biochemistry and Molecular Pharmacology, NYU Grossman School of Medicine, New York, NY 10016, USA. ${ }^{2}$ Laura and Isaac Perlmutter NYU Cancer Center and NYU Grossman School of Medicine, New York, NY 10016, USA. ${ }^{3}$ Howard Hughes Medical Institute, NYU Grossman School of Medicine, New York, NY 10016, USA. ${ }^{4}$ Institute of Enzymology, Research Centre for Natural Sciences, Budapest HU-1117, Hungary. ${ }^{5}$ Department of Microbiology, NYU Grossman School of Medicine, New York, NY 10016, USA. ${ }^{6}$ Department of Pharmacology and University of Washington, Seattle, WA 98195, USA. ${ }^{7}$ Howard Hughes Medical Institute, University of Washington, Seattle, WA 98195, USA. ${ }^{8}$ Univ Lille, INSERM, CHU Lille, UMR-S 1172, Lille Neuroscience and Cognition Research Center, F-59000 Lille, France. ${ }^{9}$ ENSCL-Centrale Lille, CS 90108 , F-59652

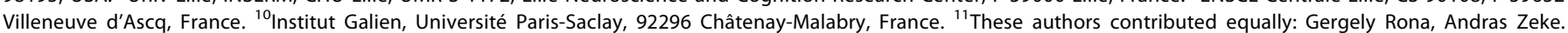
花mail: meike.dittmann@nyumc.org; michele.pagano@nyumc.org Edited by G. Melino
}

Received: 27 August 2021 Revised: 8 November 2021 Accepted: 10 November 2021 Published online: 3 December 2021 
a

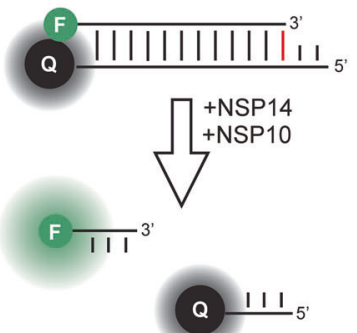

C

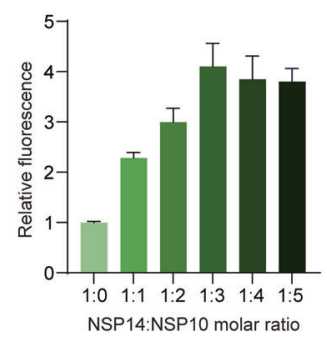

b

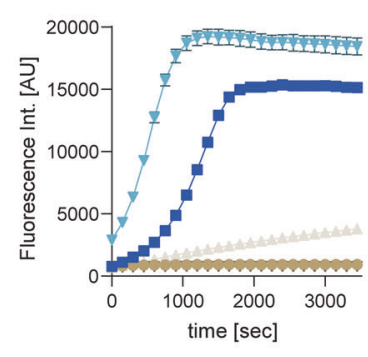

$-\mathrm{NSP} 14+\mathrm{NSP} 10$
SARS-CoV

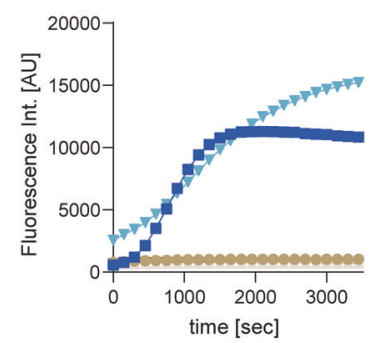

MERS-CoV

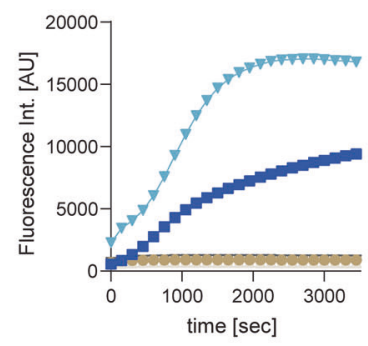

d

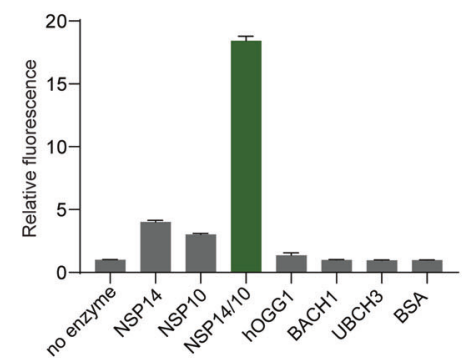

e

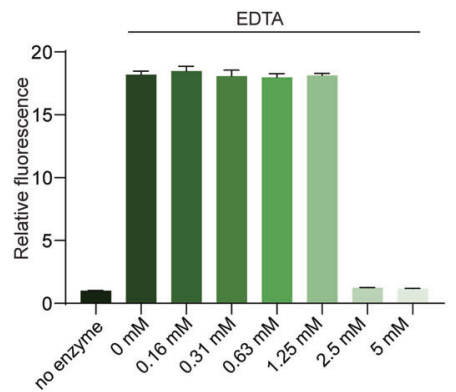

f

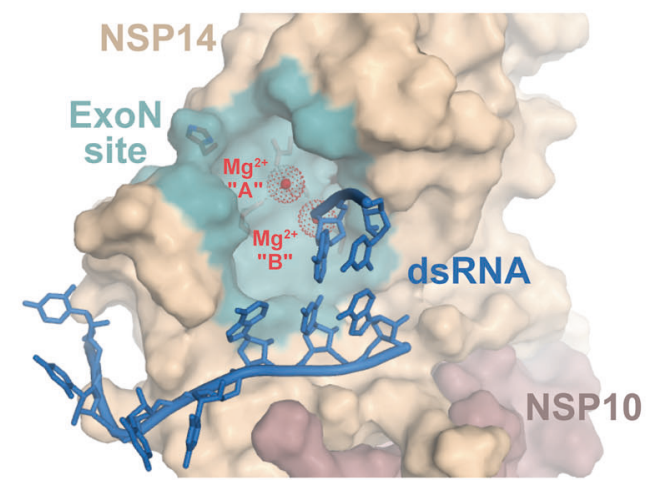

g

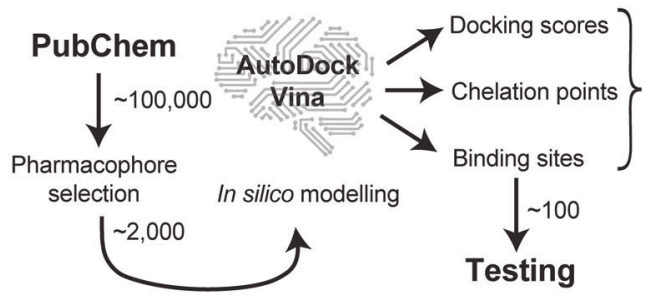

h

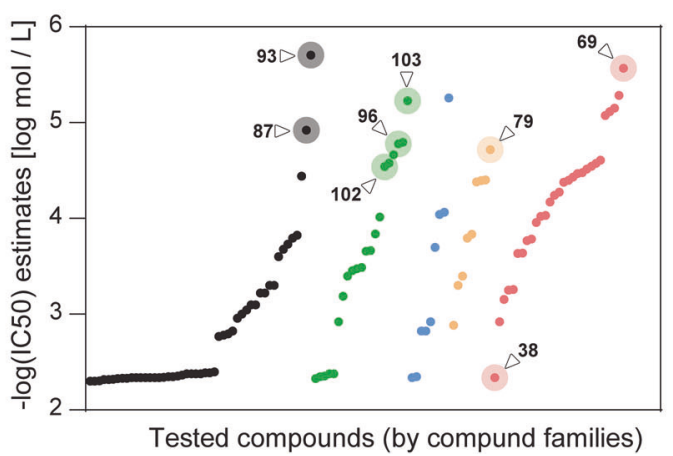

i

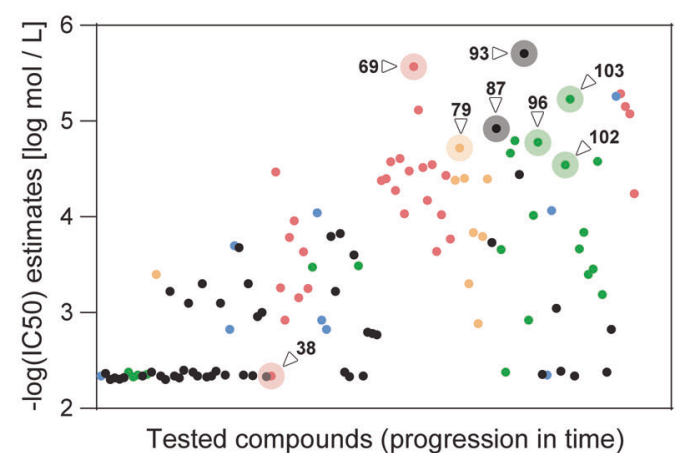

completion within 25 min (Fig. 1B and Supplementary Fig. 2B). All NSP14/10 complexes showed similar activity on the FRET dsRNA probes. In agreement with the literature, an excess of NSP10 helps NSP14 activity (Fig. 1C). So, for all downstream assays, NSP14/10 was used at a 1:3 molar ratio unless otherwise stated. The specificity of the reaction was demonstrated by the lack of signal in samples containing either NSP10 alone, NSP14/10 in the presence of EDTA (a Mg ${ }^{2+}$ chelating agent that inhibit the ExoN activity), or unrelated purified proteins (Fig. 1B and D). NSP14/10 exonuclease was inhibited by EDTA at a concentration of $\sim 2.5 \mathrm{mM}$, which can chelate free $\mathrm{Mg}^{2+}$ present in the reaction mixture at $2 \mathrm{mM}$ concentration (Fig. 1E).

The available structures of NSP14/10 of both SARS-CoV-2 and SARS-CoV display a canonical DEDDh ExoN catalytic site, which coordinates $\mathrm{Mg}^{2+}$ ions $[2,12,14,15]$. We first selected 25 compounds that are either broad-spectrum nuclease inhibitors or FDA-approved $\mathrm{Mg}^{2+}$ chelator drugs. A number of compounds including Dolutegravir, Dicoumarol, N-Hydroxy-isoquinoline-1,3-dione and, 
Fig. 1 In vitro NSP14/10 activity assay and inhibitor screening. a Schematics showing dsRNA FRET-based NSP14 exonuclease activity assay. NSP14/10 recognizes the terminal mismatch and removes bases from the $3^{\prime}$ end of the substrate strand until the two RNA strands separate and the fluorescence signal increases. b Activity curves of SARS-CoV-2, SARS-CoV and MERS-CoV NSP14/10 complex using Oligo D. RNase A was used as a positive control and NSP10 alone was used as a negative control. For all downstream assays SARS-CoV-2 NSP14/10 was used in combination with Oligo D unless otherwise stated. Data shown from one independent measurement using technical duplicates. Error bars represent SD. c Bar graph shows exonuclease activity of the NSP14:NSP10 complex at different molar ratios. Relative activity was normalized to NSP14 alone. Fluorescence was read after $1 \mathrm{~h}$ of reaction time. d Specificity of the NSP14/10 complex over a variety of purified proteins (8Oxoguanine DNA Glycosylase [OGG1]; BTB Domain and CNC Homolog 1 [Bach1]; Ubiquitin conjugating enzyme UbcH3; bovine serum albumin [BSA]). As proposed by the literature, NSP14 alone barely shows activity. Relative activity was normalized to a reaction mixture without any added purified protein. Fluorescence was read after $1 \mathrm{~h}$ of reaction time. e Bar graph shows EDTA sensitivity of the NSP14/10 exonuclease activity. Relative activity was normalized to a reaction mixture without NSP14/10. Fluorescence was read after $1 \mathrm{~h}$ of reaction time. f Structural model used for the in silico screening showing the NSP14 ExoN domain site with $\mathrm{Mg}^{2+}$ ions (red) and substrate dsRNA (blue). Surface of the ExoN catalytic site is colored light blue. Core catalytic residues are shown in stick representation. $\mathbf{g}$ Schematics of our computerassisted drug design (CADD) and heuristic in silico screening approach. $\mathbf{h} 122$ compounds and their -log(IC $\left.C_{50}\right)$ values (either exact or extrapolated) against NSP14/10 activity. Compounds listed on the X-axis are grouped and colored coded according to their various chemical families (left graph). i 122 compounds and their - $\log \left(\mathrm{IC}_{50}\right)$ values (either exact or extrapolated) against NSP14/10 activity. Compounds listed on the $X$-axis are ordered in progression over time (X-axis) and colored coded according to their various chemical families (right graph). Inhibitors were discovered using an iterative computer-assisted drug design and testing approach, leading to progressively better compounds over time.

5,5'-Methylenedisalicylic acid, showed detectable NSP14/10-inhibitory activities using our FRET assay. The results of these initial tests were instrumental to develop structural models of the NSP14 ExoN catalytic site that includes two $\mathrm{Mg}^{2+}$ ions, a substrate nucleic acid, and individual active compounds. This modeling incorporates 3 main principles: (i) both $\mathrm{Mg}^{2+}$ ions are appropriately coordinated by the enzyme and chelated by the inhibitor; (ii) NSP14 is loaded with an RNA substrate in its post-hydrolysis state; and (iii) the highly conserved ExoN site normally occupied by the last 3' nucleotide is free to be occupied by the inhibitors stacking against the rest of the RNA (Fig. 1F). Using these criteria, we filtered $\sim 100,000$ compounds and subsequently narrowed them down to a list of $\sim 2000$ compounds that are structurally compatible to the active site. These compounds were then subjected to in silico docking and scored for docking energy, chelation potential, and the possibility to bind the ExoN site (Fig. 1G). Based on the docking scores, we picked 122 compounds for in vitro testing using our FRET-based assay, which led to the identification of 23 inhibitors with an $\mathrm{IC}_{50}$ lower than $40 \mu \mathrm{M}$ (Fig. $1 \mathrm{H}, \mathrm{I}$, Supplementary Fig. 3, Supplementary Fig. 4, and Table S2). In addition to various isolated hits, we identified 4 families of compounds as NSP14 inhibitors: (1) Methylenedisalicylic acids, (2) N-hydroxy-isoquinoline-1,3-diones, (3) 2-Styrylquinoline derivatives, and (4) Chalconoids. Figure $1 \mathrm{H}$ shows the compounds grouped by families and potency, while Fig. 1 I shows the progression in identifying better compounds over time, providing a validation of our iterative computer-assisted drug design and testing approach. Importantly, the low $\mu \mathrm{M} I \mathrm{C}_{50}$ compounds (\#69, \#79, \#87, \#93, \#96, \#102, \#103) were similarly potent in inhibiting the NSP14/10 complex of both SARS-CoV and MERS-CoV (Fig. 2A, B, Supplementary Figs. 3, 4, and Table S2). While EDTA was only able to inhibit NSP14 activity when its concentration exceeded that of the free $\mathrm{Mg}^{2+}$ (Fig. 1E), the inhibitors identified here, worked at concentrations below that of free $\mathrm{Mg}^{2+}$, arguing for their ability to chelate the $\mathrm{Mg}^{2+}$ present in the catalytic pocket. We also confirmed the inhibitory potential of select compounds using gel electrophoresis as an orthogonal method (Fig. 2C and Supplementary Fig. 5A). Differential scanning fluorimetry proved that none of the tested inhibitors affected the melting temperature of the complex, suggesting that they do not work by affecting the folding of the NSP14/10 complex (Fig. 2D and Supplementary Fig. 5B). Finally, none of the tested compounds showed quenching or autofluorescence, excluding false positive and false negative hits (Supplementary Fig. 5C). Docking of our best candidates into a post-catalytic model of the NSP14-NSP10-dsRNA complex shows shared as well as unique features. One common theme is that the compounds stack against the penultimate nucleobase with a planar, aromatic ring system and coordinate the $\mathrm{Mg}^{2+}$ ions using one or more substituents. The most probable docking poses of compounds \#79 and \#96 (Fig. 1F and Fig. 2E) showed a largely overlapping binding site focused at $\mathrm{Mg}^{2+}$ "A" (the catalytic metal ion). They occupy the site of the last, cleaved nucleotide while also extending towards the mobile, catalytic His (especially with compound \#79). Compound \#102 likely binds in a different fashion, adopting a pose with a weaker stacking against the penultimate nucleotide and sandwiching inbetween the incoming RNA strand and the protein surface instead (Fig. 2E).

Next, we sought to test our compounds in viral infection assays. To this end, compounds displaying low $\mu \mathrm{M} \mathrm{IC} \mathrm{I}_{50}$ (\#69, \#79, \#87, \#93, \#96, \#102, \#103) were tested individually or in combination with remdesivir for their ability to inhibit the infection by the human seasonal $\mathrm{CoV}, \mathrm{HCoV}-\mathrm{OC} 43$, and the pandemic CoV, SARSCoV-2. To distinguish a direct effect on viral replication from an effect on the viability of the host cells, we in parallel measured the cellular toxicity of all compounds in uninfected cells. As the HCoVOC43 ExoN domain shows high sequence similarity to that of SARS-CoV2 (Supplementary Fig. 1A), the use of HCoV-OC43 allowed us to screen the top hit compounds in a BSL2 environment to evaluate pan-CoV activity. Viral spread in the presence of serial compound dilutions was evaluated by highcontent imaging and analysis as previously described (Fig. 3A) [16]. None of the compounds had detectable inhibitory activity against HCoV-OC43 on their own (Supplementary Fig. 6A, B); however, they synergized with remdesivir (Fig. 3B and Supplementary Fig. 7A-B). Compounds \#79 (7-trifluoromethyl-N-(4fluorobenzyl)-2-hydroxy-1,3-dioxo-4H-isoquinoline-4-carboxamide) [17], \#96 (Isobavachalcone), and \#102 (Sofalcone) showed the highest synergistic effect with remdesivir, lowering the $\mathrm{EC}_{50}$ values of remdesivir by $\sim 5$-fold. The results with SARS-CoV-2, performed in a BSL3 environment, closely mirrored the HCoVOC43 results. While no direct antiviral effect was detected for the drugs on their own (\#69, \#79, \#87, \#93, \#96, \#102, \#103) (Supplementary Fig. 8A-B), compounds \#79, \#96 and \#102 synergized with remdesivir (Fig. 4 and Supplementary Fig. 9A-B). This synergism is substantial considering the rather high individual $I C_{50}$ of these compounds $(\sim 30 \mu \mathrm{M})$.

\section{DISCUSSION}

By combining a novel assay measuring the catalytic activity of NSP14/10 with in silico modeling and screening, we identified a series of inhibitors of the coronavirus NSP14/NSP10 enzyme complex showing activity both in vitro and in vivo (Supplementary Fig. 9C). CoVs rely on their ExoN activity for proper propagation $[4,5]$, which is evident when measured over many replication 
a<smiles>CC(C)=CCOc1ccc(CC=C(c2ccc(C(=O)C=Cc3ccc(O)cc3)cc2)c2ccc(OCC=C(C)C)c(CC=C(C)C)c2O)cc1</smiles>

b
C

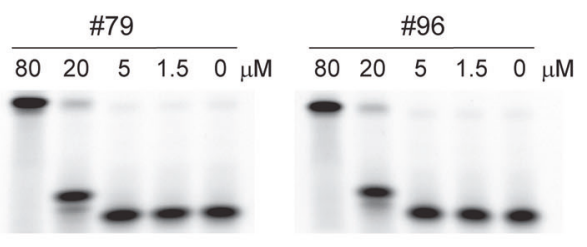

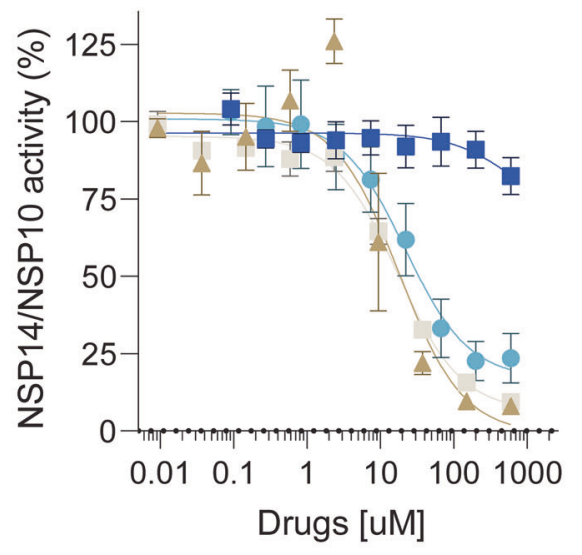

- \#38

$-\# 79$

-1- \#96

- \#102

$\# 79 I C_{50}=19.43 \pm 8.37$ uM

\#96 IC $\mathrm{IO}_{50}=17.43 \pm 1.39 \mathrm{uM}$

$\# 102$ IC $_{50}=21.99 \pm 6.02 \mathrm{uM}$ d

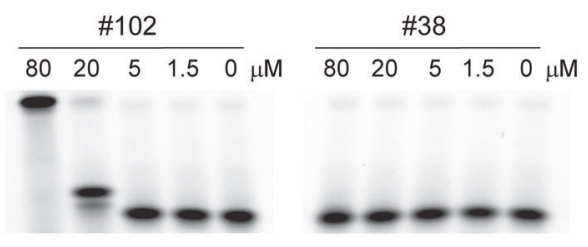

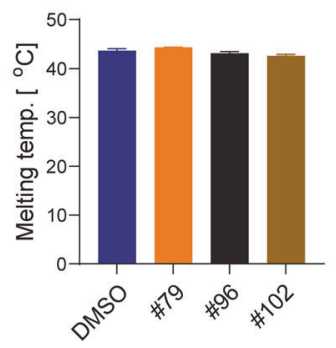

e

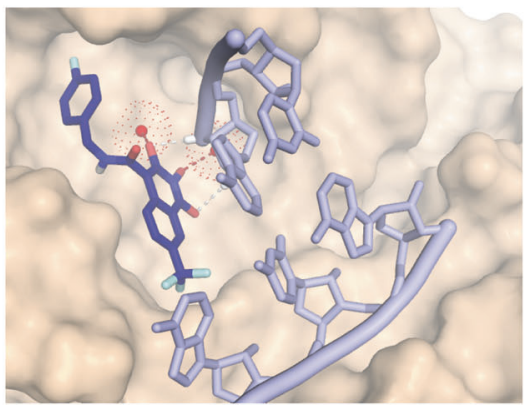

\#79

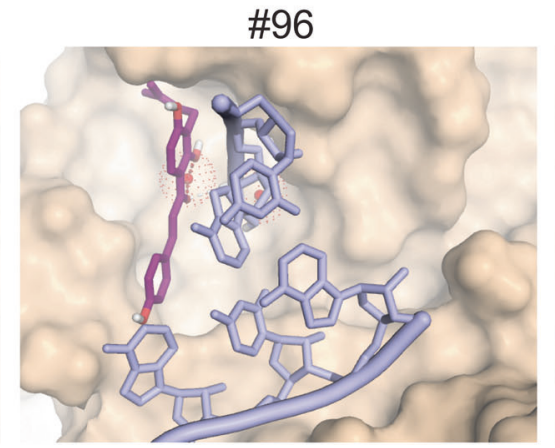

\#102

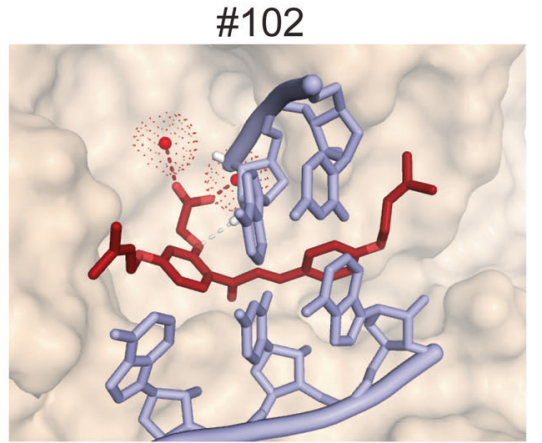

Fig. 2 Characterization of novel NSP14/10 inhibitors. a Chemical structures of the three compounds (\#79, \#96 and \#102) that performed the best in the viral infection assays. Compound \#38, which does not inhibit NSP14, was included in downstream assays as a negative control. b Dose-response curves of NSP14/10 activity in the presence of compounds \#38 (negative control), \#79, \#96 and \#102. Average IC 50 values were calculated from three independent measurements using technical duplicates. Error bars represent SD. c A gel-based assay was used to verify inhibitory potential of compounds \#79, \#96, \#102, and \#38. Inhibition of the NSP14/10 exonuclease activity results in the reduction of the full-length dsRNA oligo and increase in faster migrating bands. Compounds were used at the indicated concentrations. d Differential scanning fluorimetry performed in the presence or absence of the indicated compounds at $50 \mu \mathrm{M}$ concentrations. Graph shows calculated mean melting temperatures of the NSP14/10 complex in the presence of the indicated compounds. Error bars represent SD. e Docking poses of compounds, predicted to stack either against the last nucleobase (\#79, \#96) or under the RNA (\#102) while simultaneously engaging the $\mathrm{Mg}^{2+}$ ion(s) at the catalytic site (red dots).

cycles. The temporal limitation of our assay only allows up to a maximum of 2-3 replication cycles, which may explain why our inhibitors do not show activity on their own. In contrast, the inhibitors display sensitization of viral replication to remdesivir, providing an important proof of concept for a new therapeutic approach against CoVs. Ribonucleotide analogs working as chain terminators do not induce lethal mutagenesis and can be subdivided into direct or delayed terminators. Direct chain terminators are incorporated at the end of RNA strand without being followed by other ribonucleotides. Therefore, they are immediately sensed by the RNA-dependent RNA polymerase (NSP12) and by NSP14, and can be removed from the viral genome in a single step. Remdesivir's incorporation is instead followed by $\sim 3$ ribonucleotides, acting as a delayed chain terminator [18-20]. We believe that remdesivir incorporated into nascent RNA is an excellent target for NSP14 ExoN inhibitory drugs since multiple ribonucleotides need to be removed until remdesivir can be excised from the viral genome [21]. This provides a longer window of opportunity for the inhibitors to act on NSP14.

When evaluating our compounds, one must note that the degree of antiviral efficacy and synergy with remdesivir is subject to a multitude of biological factors influencing drug uptake and availability. Of our top hits displaying synergy with remdesivir, 
a

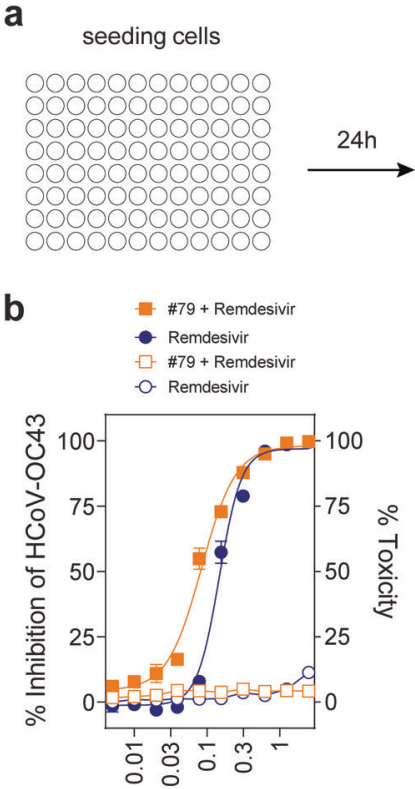

78 nM Remdesivir
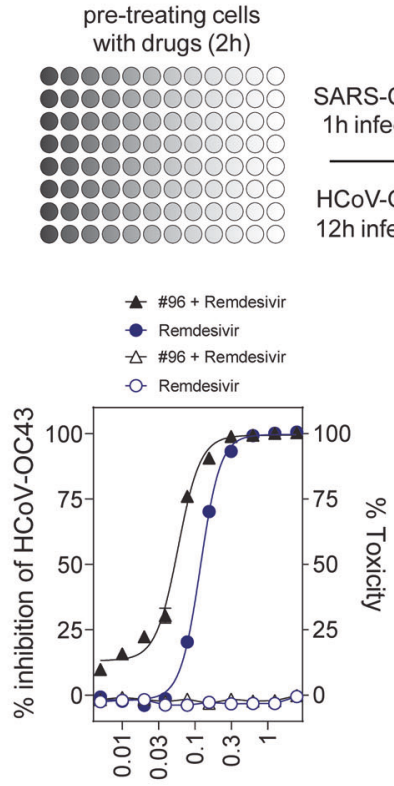

78 nM Remdesivir

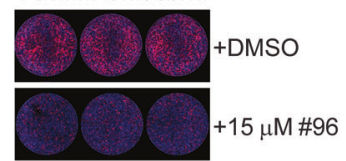

remove virus and

re-adding drugs

SARS-CoV-2

$1 \mathrm{~h}$ infection

HCoV-OC43

$12 \mathrm{~h}$ infection

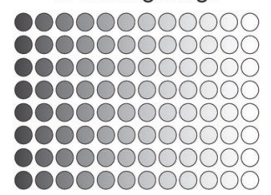

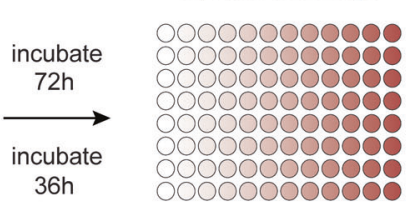

fix, stain and image

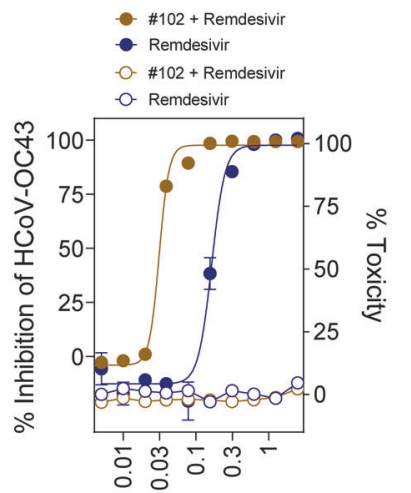

78 nM Remdesivir

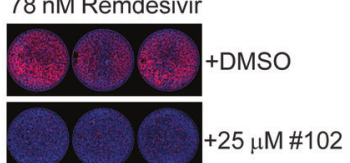

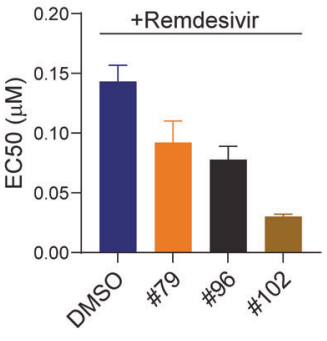

Fig. 3 Synergistic effect of select compounds with remdesivir using HCoV-OC43 viral infection assay. a Schematics showing the viral infection assay workflow. Drug treated cells were infected with HCoV-OC43 or SARS-CoV-2, fixed, stained, and imaged at indicated times post infection. Cytotoxicity was measured in similarly drug-treated uninfected cells using either the CellTiter-Glo or the alamarBlue assay. b Representative graphs are shown of the antiviral activity (full symbols) and cytotoxicity (empty symbols) of compounds \#79, \#96 and \#102 in $\mathrm{HCM} 3$ cells infected with HCoV-OC43. Compounds were applied at the following concentrations: \#79: $60 \mu \mathrm{M}$, \#96: 15 $\mu \mathrm{M}$, \#102: $25 \mu \mathrm{M}$. Remdesivir concentrations are indicated on the X-axis in $\mu \mathrm{M}$. Error bars represents SEM. IF images of representative wells show anti-N staining (red) and DAPI signal (blue) at indicated drug concentrations. Graph shows the $\mathrm{EC}_{50}$ values from three independent experiments using technical triplicates. Error bars represents SEM.

isobavachalcone (\#96) is extracted from medicinal herbs [22]; compound \#79 was originally synthesized as an early-stage antiHIV agent [23]; and sofalcone, albeit not FDA-approved, has been demonstrated by clinical studies in Japan to be well tolerated by humans for the treatment of gastric diseases [24, 25]. Optimization of these hits has the potential to develop them into potent panCoV therapeutics (Fig. 5).

\section{MATERIALS AND METHODS}

\section{Development of the dsRNA-loaded NSP10-NSP14 model}

Based on the nearly 100\% sequence homology around the ExoN catalytic site of SARS-CoV-2 and SARS-CoV, we used SARS-CoV NSP14 crystal structures in our modeling. Several published structures are available (5C8T, $5 \mathrm{C} 8 \mathrm{U}$ and $5 \mathrm{C} 8 \mathrm{~S}$, with one $\mathrm{Mg}^{2+}$ ion $\left(\mathrm{Mg}^{2+}\right.$ "B") at the catalytic site). The structure, 5NFY, was taken as our starting template, due to its decent electron density map. Although structure 5NFY contains no explicit $\mathrm{Mg}^{2+}$ ions, the octahedral coordination of a single density near the catalytic site $\left(\mathrm{H}_{2} \mathrm{O}\right.$ in the PDB file) allowed its re-interpretation as the metal ion $\mathrm{Mg}^{2+}$ " $B$ ". The predicted site of the other catalytic metal ion, $\mathrm{Mg}^{2+}$ " $\mathrm{A}$ " contained no matching electron density in the published NSP14 structures and was therefore determined to be a highly conserved metal ion binding site which is only occupied in the full, substrate-bound enzyme. Structures were adjusted using Coot (version 0.8.6.1) and PyMol (version 1.8), and protonated on the MolProbity webserver (including His, Gln and Asn ring flips). The protein models were prepared for docking using AutoDockTools (version 1.5.7), removing $\mathrm{Zn}^{2+}$ ions, merging non-polar hydrogens and adding Kollman charges. $\mathrm{Mg}^{2+}$ ions, that have no default charge under the Kollman system, were manually assigned a charge +2.0 . We did not to use fractional charges, to offset the lack of chelation energies under AutoDock. Although derived from SARS-CoV, the final model also matched well with published SARS-CoV-2 NSP14 structures (PDB entries 7MC5, 7MC6 and 7DIY).
To model the RNA-bound NSP14-NSP10 complex, we utilized the structure of the very distantly homologous TREX1 as a guide, loaded with dsDNA (PDB: 4YNQ). The position of the substrate in this model is also very similar to other DEDD exonucleases-such as human ERI1 (PDB: 4QOZ), yeast Pan2 (PDB: 6R9J) exonucleases or the Lassa virus nucleoprotein (PDB: $4 \mathrm{FVU}$ ). The dsRNA in our model was adjusted to maximize $\mathrm{H}$-bonding with the catalytic site of ExoN (using PyMol and Coot), and the last nucleotide positioned into the catalytic site, with $\mathrm{Mg}^{2+}$ ions at a canonical phosphate coordinating position. The RNA strands were also intentionally slightly distorted (in Coot), to mimic a mismatching $3^{\prime}$ end. Our resulting model was very similar to those determined experimentally later, including the apo NSP14 (PDB: 7MC6) and substrate-loaded NSP14 complexes (PDB: $7 \mathrm{NOB}$ ). Then the last nucleotide was removed from the final complex (with its phosphate). We argued that any compound that could stabilize this post-catalytic state (that is also pre-catalytic if the dsRNA does slide into place with one position off) would be an effective inhibitor. Then we prepared this model for docking using MolProbity, PyMol and AutoDockTools as outlined previously. Compounds docking into this model with high scores usually show at least some inhibition in our assay, confirming our approach.

\section{Selection of candidate compounds}

The very initial set of compounds was selected from a pool of pan-enzyme inhibitors and FDA-approved drugs known to act on viral endonucleases, exonucleases or integrases, without any docking simulations. Out of this pool only a few candidates showed inhibitory potency. Because these molecules can adopt a pose that avoids half of the catalytic site (where the RNA is proposed to enter), we concluded that the RNA must be present in the model to yield meaningful results. In the subsequent rounds, we utilized the fully dsRNA-loaded (post-catalytic) complex to successfully identify several potent inhibitors. Candidates for docking were selected from publicly available and commercial databases (PubChem, ZINC, Mcule, MolPort, ChemFaces, etc.) containing "drug-like" candidates, with sufficient 

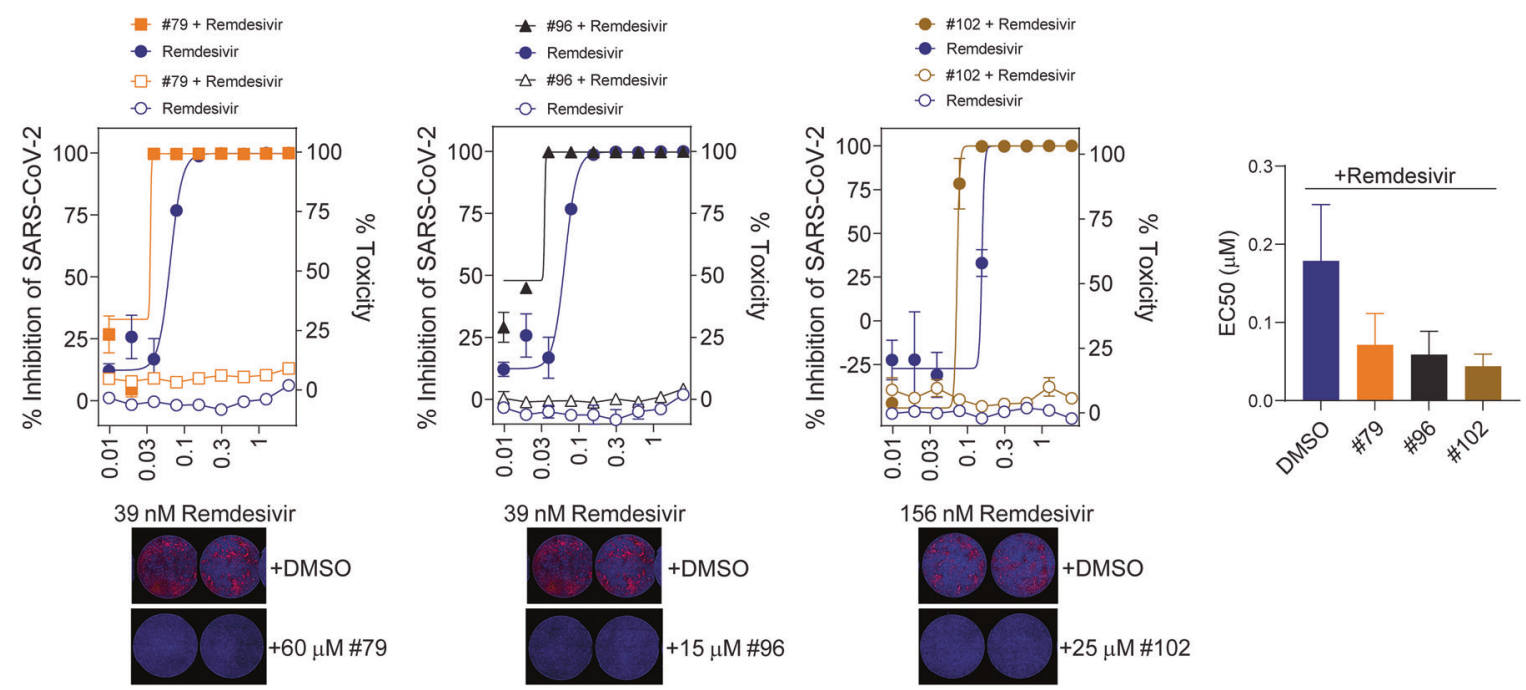

Fig. 4 Synergistic effect of select compounds with remdesivir using SARS-CoV-2 viral infection assay. Representative graphs are shown of the antiviral activity (full symbols) and cytotoxicity (empty symbols) of compounds \#79, \#96 and \#102 in A549 ${ }^{+A C E 2}$ cells infected with SARSCoV-2. Compounds were applied at the following concentrations: \#79: $60 \mu \mathrm{M}$, \#96: $15 \mu \mathrm{M}$, \#102: $25 \mu \mathrm{M}$. Remdesivir concentrations are indicated on the X-axis in $\mu \mathrm{M}$. Error bars represents SEM. IF images of representative wells show anti-N staining (red) and DAPI signal (blue) at indicated drug concentrations. Graph shows the $\mathrm{EC}_{50}$ values from three independent experiments using technical triplicates. Error bars represents SEM.

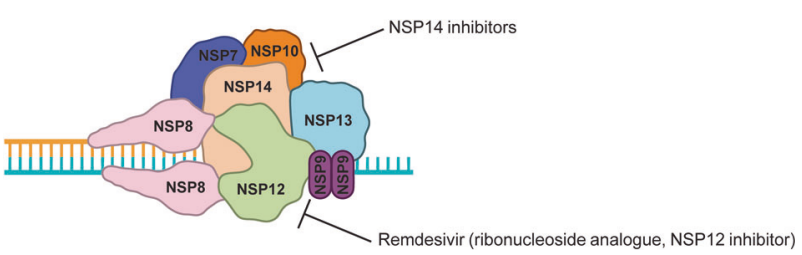

Fig. 5 NSP14/10 inhibition as a therapeutic approach. Schematics showing our therapeutic approach where inhibiting NSP14 potentiates the inhibition of NSP12 (with remdesivir, a ribonucleoside analog).

structural diversity. Once the very first hits were uncovered, we analyzed their structures and used them in subsequent database queries. In addition, new heuristically designed molecular architectures were introduced at each run to ensure scaffold diversity. We performed this method iteratively, until it converged on several potently inhibiting compound families.

\section{Docking of ligands}

The computationally predicted 3D structure of candidate compounds was downloaded from PubChem. Whenever no structure was available in PubChem, we used either the OpenBabel or MolView websites to generate 3D molecular coordinates in a suitable format. Structures were then individually reviewed in PyMol, setting the predicted protonation state at $\mathrm{pH}=7.0$ and correcting their geometries when necessary. Reviewed molecules were converted into pdbqt files using AutoDockTools (adding Gasteiger charges). Special care was taken when setting the torsion tree in case of compounds with conjugated double bonds or aromatic systems, to avoid chemically implausible docking poses. Compounds were then docked into the NSP14/10 models using AutoDock Vina (version 1.1.2), with a fully rigid protocol, and a target box limited around the ExoN catalytic site (to save computation time and facilitate evaluation).

\section{Plasmid constructs}

The CDNA encoding NSP14 and NSP10 of SARS-CoV-2, SARS-CoV, and MERS-CoV were codon optimized for expression in $E$. coli using the codon optimization tool of GenScript. Custom CDNAs were synthesized by GenScript and subcloned into a pET-30a(+) vector backbone, resulting in a C-terminal 6X His-tag following a flexible linker (GGGSGGGS) for all the constructs.

\section{Expression and purification of NSP14/10}

NSP14 and NSP10 proteins of SARS-CoV-2, SARS-CoV and MERS-CoV were either purified in house or by GenScript. In brief, vectors encoding NSP14 and NSP10 were transformed into OverExpress C41(DE3) or BL21(DE3) E. coli. $5 \mathrm{~L}$ of $E$. coli were grown at $37^{\circ} \mathrm{C}$ to an $\mathrm{OD}_{600}$ of 0.6 , induced with $1 \mathrm{mM}$ IPTG and shaken at $15^{\circ} \mathrm{C}$ for $16 \mathrm{~h}$. Cells were harvested and pelleted by centrifugation at $4000 \times \mathrm{g}$ for $30 \mathrm{~min}$ at $4^{\circ} \mathrm{C}$. Cell pellets were resuspended in lysis buffer $(50 \mathrm{mM}$ TRIS- $\mathrm{HCl} \mathrm{pH}=8.0,150 \mathrm{mM} \mathrm{NaCl}, 0.5 \%$ Triton X-100, $5 \mathrm{mM} \beta$-mercaptoethanol), lysed by sonication and clarified using centrifugation $\left(30000 \times \mathrm{g}, 30 \mathrm{~min}\right.$ at $\left.4^{\circ} \mathrm{C}\right)$. Clarified lysate was supplemented with $20 \mathrm{mM}$ imidazole and incubated with Ni-NTA resin (Qiagen) $4{ }^{\circ} \mathrm{C}$ for $1 \mathrm{~h}$, washed with wash buffer $(50 \mathrm{mM}$ TRIS- $\mathrm{HCl} \mathrm{pH}=8.0$, $300 \mathrm{mM} \mathrm{NaCl}, 5 \%$ glycerol, $40 \mathrm{mM}$ imidazole and $5 \mathrm{mM} \beta$-mercaptoethanol), and eluted with elution buffer ( $50 \mathrm{mM}$ TRIS-HCl pH=8.0, $100 \mathrm{mM} \mathrm{NaCl}$, $350 \mathrm{mM}$ imidazole, $5 \%$ glycerol and $5 \mathrm{mM} \beta$-mercaptoethanol). Eluate was

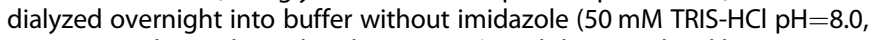
$150 \mathrm{mM} \mathrm{NaCl}, 5 \%$ glycerol and $1 \mathrm{mM} \mathrm{DTT}$ ), and then sterilized by a $0.22 \mu \mathrm{m}$ filter. Protein concentration was determined by Bradford protein assay with BSA as a standard and concentrated as necessary. Samples were then aliquoted and stored at $-80^{\circ} \mathrm{C}$.

\section{FRET exonuclease activity assay}

To prepare the dsRNA substrates, RNA oligos were annealed at a final concentration of $50 \mu \mathrm{M}$. To anneal the ssRNA oligos, samples were heated in a PCR cycler to $95^{\circ} \mathrm{C}$ for $5 \mathrm{~min}$ and then cooled to $5^{\circ} \mathrm{C}$ in $5^{\circ} \mathrm{C}$ increments over 18 cycles of $1 \mathrm{~min}$ each. Exonuclease activity assays were performed at $37^{\circ} \mathrm{C}$ in black bottom 96 well plates. The reactions were performed in the following buffer: $50 \mathrm{mM}$ TRIS- $\mathrm{HCl} \mathrm{pH} 7.5,2 \mathrm{mM} \mathrm{MgCl} 2,2 \mathrm{mM}$ DTT. NSP14 and NSP10 were used at $200 \mathrm{nM}$ and $600 \mathrm{nM}$, respectively, maintaining a molar ratio of $1: 3$, while the dsRNA substrate was added at a final concentration of $1 \mu \mathrm{M}$. The fluorescence intensity of each well was measured every $150 \mathrm{~s}$ on an Infinite 200 PRO microplate reader (Tecan) over the course of the activity assay using the following settings: Excitation $490 \mathrm{~nm}( \pm 9 \mathrm{~nm}) /$ Emission $520 \mathrm{~nm}( \pm 20 \mathrm{~nm})$. Activity measurements for MERS-CoV were performed identically to SARS-CoV-2. Activity assays of SARS-CoV NSP14/NSP10 were performed at concentrations $40 \mathrm{nM}$ and 120 $\mathrm{nM}$, respectively.

\section{$I_{50}$ calculation}

Selected inhibitors were serial diluted in the indicated range. The fluorescence of each dilution was measured in the linear activity range and used to determine the $\mathrm{IC}_{50}$ for selected compounds using the threeparameter non-linear regression function of GraphPad Prism (9.1.2). 
Activity of inhibited NSP14/10 was normalized to the control sample with the vehicle control.

\section{Compound screening}

Screening was performed using a custom compound selection originating from commercial sources (Sigma, Selleck, MedChem Express, Tocris, Enamine, MCule, Key Organics, Hit2Lead, Vitas-M Laboratory and ChemFaces) as well as custom synthesized compounds from the Styrylquinoline and NHID groups, with each compound having an average purity of $95 \%$. Compounds were reconstituted at $100 \mathrm{mM}$ in DMSO, aliquoted and stored under argon gas at $-80^{\circ} \mathrm{C}$. Primary screening was performed at 500 or $250 \mu \mathrm{M}$ compound concentration, where compounds were pre-incubated with the NSP14/10 complex for $15 \mathrm{~min}$ at room temperature before adding the FRET substrate. Secondary screenings on select compounds were performed at 100 or $50 \mu \mathrm{M}$. To exclude false positive and negative hits, compounds at $250 \mu \mathrm{M}$ were tested for autofluorescence and quenching. Compounds were color coded based on their chemical scaffold grouping. They were either ordered on the $\mathrm{X}$-axis based on their chemical grouping (Fig. 1E) or in progression over time, (Supplementary Fig. 2F). For each compound tested, we plotted its $-\log \left(I_{50}\right)$ value on the Y-axis (either exact or extrapolated value).

\section{Exonuclease gel assay}

6FAM labeled RNA oligos were annealed as described previously. The enzymatic reactions were performed as described in the FRET kinetic exonuclease activity assay but using $5 \mu \mathrm{M}$ dsRNA substrate in a total volume of $20 \mu \mathrm{L}$. After $45 \mathrm{~min}$, reactions were stopped with $\mathrm{Novex}^{\mathrm{TM}} \mathrm{Hi}-$ Density TBE Sample Buffer containing EDTA (5 mM), and chilled on ice. 10 $\mu \mathrm{L}$ of samples were loaded into a $20 \%$ TBE Gel and run at $200 \mathrm{~V}$ for $80 \mathrm{~min}$. Gels were imaged using a BIO-RAD Gel Doc ${ }^{\mathrm{TM}} \mathrm{XR}+$ imager.

\section{Differential scanning fluorimetry}

Melting temperature of the NSP14/10 complex in the presence or absence of select compounds were assayed by differential scanning fluorimetry using either Protein Thermal Shift ${ }^{\mathrm{TM}}$ (Thermo Scientific) or GloMelt ${ }^{\mathrm{TM}}$ Thermal Shift Protein Stability Kit (Biotium) according to the manufacturer's protocol. Assays were performed in a 96-well qPCR plate in a final volume of $25 \mu \mathrm{L}$ in the following reaction buffer: $50 \mathrm{mM}$ TRIS- $\mathrm{HCl} \mathrm{pH}=7.5$ $2 \mathrm{mM} \mathrm{MgCl}_{2}, 2 \mathrm{mM}$ DTT. NSP14 and NSP10 were allowed to pre-complex at an equimolar ratio of $5 \mu \mathrm{M}$ at RT for 15 min before adding the compounds at $50 \mu \mathrm{M}$ final concentration. The temperature was linearly increased in a QuantStudio3 Real-Time PCR System with a step of $0.05^{\circ} \mathrm{C} / \mathrm{s}$, from $25^{\circ} \mathrm{C}$ to $95^{\circ} \mathrm{C}$, and fluorescence readings were taken at each interval (Protein Thermal Shift Assay: ROX filter set; GloMelt Thermal Shift Assay: FAM filter set). Melting temperatures were calculated as the inflection point of the melting curve using the derivative analysis function of the QuantStudio ${ }^{\mathrm{TM}}$ Design \& Analysis Software (version 1.4.3).

\section{Cell lines and viruses}

Cell lines were purchased from ATCC. A549 ${ }^{+ \text {ACE2 }}$ cells, were created as described previously [16]. A549+ACE2, HMC3 (ATCC CRL-3304) and MRC-5 (ATCC CCL-171) cells were maintained in Dulbecco's modified Eagle medium (DMEM) supplemented with $10 \%$ fetal bovine serum (FBS), $1 \%$ penicillin/streptomycin and incubated at $37{ }^{\circ} \mathrm{C}$ under $5 \% \mathrm{CO}_{2}$. Cell lines were routinely monitored for mycoplasma contamination using the Universal Mycoplasma Detection Kit (ATCC 30-1012 K). All cells used in this study tested negative for Mycoplasma contamination. A549 ${ }^{+A C E 2}$ cells were used for SARS-CoV-2 infection assays. SARS-CoV-2 isolate USA-WA1/ 2020 stocks were prepared as described previously [16]. HMC3 cells were used for HCoV-OC43 infection assays. HCoV-OC43 viral stocks (ATCC VR1558) were propagated/isolated as detailed below. MRC-5 cells were seeded at a density of $2 \times 10^{6}$ cells in $100 \mathrm{~mm}$ dish. The next day, the cells were infected with $3 \times 10^{6} \mathrm{pfu} / \mathrm{ml}$ of HCoV-OC43 and incubated at $33^{\circ} \mathrm{C}$ for four days until $90-100 \%$ cells have cytopathic effects. The culture supernatant and infected cells were harvested, centrifuged at $1000 \times g$ for $5 \mathrm{~min}$ and the supernatant was stored at $-80^{\circ} \mathrm{C}$.

\section{SARS-CoV-2 viral infectivity assay}

A549+ACE2 cells were seeded into black 96 -well plates at $90 \%$ confluency. The next day, media was removed and replaced with complete media containing compounds/carrier $2 \mathrm{~h}$ prior to infection. Cells were then infected to reach $80-90 \%$ infected cells after $72 \mathrm{~h}$ at $37^{\circ} \mathrm{C}$. One hour post virus addition, virus was removed, and media containing compounds/ carrier was added. At $72 \mathrm{~h}$ post infection, cells were fixed by submerging in $10 \%$ formalin solution for 30-45 min. After fixation cells were washed once with $\mathrm{H}_{2} \mathrm{O}$ to remove excess formalin. Plates were dried and PBS was added to each well before exiting the BSL-3 facility. Fixed cells were permeabilized and stained with mouse monoclonal SARS-CoV anti-N antibody 1C7, which cross-reacts with SARS-CoV-2 N (kind gift of Thomas Moran), goat anti-mouse AlexaFluor 647 and DAPI. For determination of cytotoxicity, A549 ${ }^{+A C E 2}$ cells were seeded into opaque white wall 96-well plates. The following day, media was removed, replaced with media containing compounds/carrier and incubated for $72 \mathrm{~h}$. At these timepoints, ATP levels were determined by CellTiter-Glo 2.0 (Promega) using a BioTek Synergy HTX multi-mode reader.

\section{HCoV-OC43 viral infectivity assay}

HMC3 cells were seeded into black 96 -well plates at $90 \%$ confluency. The next day, media was removed and replaced with complete media containing compounds/carrier $2 \mathrm{~h}$ prior to infection. After that, $5 \mu \mathrm{l}$ of $\mathrm{HCoV}-\mathrm{OC} 43$ virus was added to each well at an $\mathrm{MOI}$ of 0.005 for a final total volume of $100 \mu \mathrm{l} /$ well. The plates were incubated at $33^{\circ} \mathrm{C}$. The viral suspension was removed after $12 \mathrm{~h}$ and drugs were added back to the cells to further incubate at $33^{\circ} \mathrm{C}$ for $36 \mathrm{~h}$. At $48 \mathrm{~h}$ post infection, cells were fixed with $4 \%$ PFA in the media for 15 min. Residual PFA was quenched with a $50 \mathrm{mM} \mathrm{NH}_{4} \mathrm{Cl}$ and $100 \mathrm{mM} \mathrm{NaCl}$ solution. Cells were permeabilized and blocked in 5\% FBS, 3\% BSA, 0.05\% Triton X-100 and $0.005 \% \mathrm{NaN}_{3}$ in PBS for an hour. Anti-N antibody (Sigma 542-7D) was diluted in 4\% FBS in PBS at 1:1000 dilution and was applied at $4{ }^{\circ} \mathrm{C}$ for $16 \mathrm{~h}$. After several washing steps, cells were stained with AlexaFluor 594 and DAPI. For determination of cytotoxicity, HMC3 cells were seeded into opaque white wall 96-well plates. The following day, media was removed, replaced with media containing compounds/carrier and incubated for $48 \mathrm{~h}$. After $48 \mathrm{~h}$, cell viability was determined using alamarBlue ${ }^{T M}$ Cell Viability Reagent using an Infinite 200 PRO microplate reader (Tecan).

\section{Imaging analysis}

Plates were scanned on a Citation 5 Imaging Multi-Mode Reader (BioTek). Gen5 software (version 3.10) was used for both image acquisition and analysis. A total number of 16 images/well were collected at $4 x$ magnification to span the entire well. DAPI channel was used to build primary masks for the nucleus. Secondary masks were built within a $15 \mu \mathrm{m}$ ring around the primary nuclear mask. Infection efficiencies were calculated from the ratio of the total fluorescence intensity within the secondary mask and the total area of the secondary mask for each well. Microscope images were prepared for publication using ImageJ (version 1.53i).

\section{Antibodies}

Antibodies used in the study were the following:

- SARS-CoV anti-N antibody 1C7, mouse monoclonal, cross-reacts with SARSCoV-2 N, kind gift of Thomas Moran, dilution: 1:1000

- HCoV-OC43 anti-N antibody, mouse monoclonal, Sigma-Aldrich, cat. no.: MAB9013, lot no.: 3587234, dilution: 1:1000

Goat anti-Mouse IgG $(\mathrm{H}+\mathrm{L})$ Highly Cross-Adsorbed Secondary Antibody, Alexa Fluor Plus 594, cat. no.: A32742, lot no.: VL316327, dilution: 1:1000

Goat anti-Mouse IgG $(\mathrm{H}+\mathrm{L})$ Highly Cross-Adsorbed Secondary Antibody, Alexa Fluor Plus 647, cat. no.: A32728, lot no.: VH311610, dilution: 1:1000

\section{DATA AVAILABILITY}

All accession codes, unique identifiers, and web links for publicly available datasets are available within the article and the reporting summary. All original data are available from the corresponding authors upon request.

\section{REFERENCES}

1. Bouvet M, Imbert I, Subissi L, Gluais L, Canard B, Decroly E. RNA 3'-end mismatch excision by the severe acute respiratory syndrome coronavirus nonstructural protein nsp10/nsp14 exoribonuclease complex. Proc Natl Acad Sci USA. 2012;109:9372-7.

2. Lin S, Chen H, Chen Z, Yang F, Ye F, Zheng Y, et al. Crystal structure of SARS-CoV-2 nsp10 bound to nsp14-ExoN domain reveals an exoribonuclease with both structural and functional integrity. Nucleic Acids Res. 2021;49:5382-92. 
3. Ma Z, Pourfarjam Y, Kim IK. Reconstitution and functional characterization of SARS-CoV-2 proofreading complex. Protein Expr Purif. 2021;185:105894.

4. Ogando NS, Ferron F, Decroly E, Canard B, Posthuma CC, Snijder EJ. The curious case of the nidovirus exoribonuclease: its role in rna synthesis and replication fidelity. Front Microbiol. 2019;10:1813.

5. Ogando NS, Zevenhoven-Dobbe JC, van der Meer Y, Bredenbeek PJ, Posthuma CC, Snijder EJ The enzymatic activity of the nsp14 exoribonuclease is critical for replication of MERS-CoV and SARS-CoV-2. J Virol. 2020;94:e01246-20.

6. Agostini ML, Andres EL, Sims AC, Graham RL, Sheahan TP, Lu X, et al. Coronavirus susceptibility to the antiviral remdesivir (GS-5734) is mediated by the viral polymerase and the proofreading exoribonuclease. mBio. 2018, 9.

7. Sheahan TP, Sims AC, Zhou S, Graham RL, Pruijssers AJ, Agostini ML, et al. An orally bioavailable broad-spectrum antiviral inhibits SARS-CoV-2 in human airway epithelial cell cultures and multiple coronaviruses in mice. Sci Transl Med. 2020;12:eabb5883.

8. Deng X, StJohn SE, Osswald HL, O'Brien A, Banach BS, Sleeman K, et al. Coronaviruses resistant to a $3 C$-like protease inhibitor are attenuated for replication and pathogenesis, revealing a low genetic barrier but high fitness cost of resistance. J Virol. 2014;88:11886-98.

9. Agostini ML, Pruijssers AJ, Chappell JD, Gribble J, Lu X, Andres EL, et al. Smallmolecule antiviral beta-d-N (4)-hydroxycytidine inhibits a proofreadingintact coronavirus with a high genetic barrier to resistance. J Virol. 2019;93: e01348-19.

10. Robson F, Khan KS, Le TK, Paris $C$, Demirbag $S$, Barfuss $P$, et al. Coronavirus RNA proofreading: molecular basis and therapeutic targeting. Mol Cell. 2020;79:710-27

11. Smith EC, Blanc H, Surdel MC, Vignuzzi M, Denison MR. Coronaviruses lacking exoribonuclease activity are susceptible to lethal mutagenesis: evidence for proofreading and potential therapeutics. PLoS Pathog. 2013;9:e1003565.

12. Ferron $F$, Subissi $L$, Silveira De Morais AT, Le NTT, Sevajol M, Gluais L, et al. Structural and molecular basis of mismatch correction and ribavirin excision from coronavirus RNA. Proc Natl Acad Sci USA. 2018;115:E162-E171.

13. Scholle MD, Liu C, Deval J, Gurard-Levin ZA. Label-free screening of SARS-CoV-2 NSP14 exonuclease activity using SAMDI mass spectrometry. SLAS Disco. 2021;26:766-74.

14. Ma Y, Wu L, Shaw N, Gao Y, Wang J, Sun Y, et al. Structural basis and functional analysis of the SARS coronavirus nsp14-nsp10 complex. Proc Natl Acad Sci USA. 2015;112:9436-41.

15. Yan L, Yang Y, Li M, Zhang Y, Zheng L, Ge J, et al. Coupling of N7methyltransferase and $3^{\prime}-5^{\prime}$ exoribonuclease with SARS-CoV-2 polymerase reveals mechanisms for capping and proofreading. Cell. 2021;84:3474-85.

16. de Vries M, Mohamed AS, Prescott RA, Valero-Jimenez AM, Desvignes L, O'Connor $\mathrm{R}$, et al. A comparative analysis of SARS-CoV-2 antivirals characterizes $3 \mathrm{CL}$ (pro) inhibitor PF-00835231 as a potential new treatment for COVID-19. J Virol. 2021;95:e01819-20.

17. Suchaud V, Bailly F, Lion C, Calmels C, Andreola ML, Christ F, et al. Investigation of a novel series of 2-hydroxyisoquinoline-1,3(2H,4H)-diones as human immunodeficiency virus type 1 integrase inhibitors. J Med Chem. 2014;57:4640-60.

18. Kokic G, Hillen HS, Tegunov D, Dienemann C, Seitz F, Schmitzova J, et al. Mechanism of SARS-CoV-2 polymerase stalling by remdesivir. Nat Commun. 2021;12:279.

19. Shannon A, Le NT, Selisko B, Eydoux C, Alvarez K, Guillemot JC, et al. Remdesivir and SARS-CoV-2: Structural requirements at both nsp12 RdRp and nsp14 Exonuclease active-sites. Antivir Res. 2020;178:104793.

20. Yin W, Mao C, Luan X, Shen DD, Shen $Q$, Su H, et al. Structural basis for inhibition of the RNA-dependent RNA polymerase from SARS-CoV-2 by remdesivir. Science. 2020;368:1499-504.

21. Gordon CJ, Tchesnokov EP, Woolner E, Perry JK, Feng JY, Porter DP, et al. Remdesivir is a direct-acting antiviral that inhibits RNA-dependent RNA polymerase from severe acute respiratory syndrome coronavirus 2 with high potency. J Biol Chem. 2020;295:6785-97.

22. Wang M, Lin L, Lu JJ, Chen X. Pharmacological review of isobavachalcone, a naturally occurring chalcone. Pharm Res. 2021;165:105483.

23. Desimmie BA, Demeulemeester J, Suchaud V, Taltynov O, Billamboz M, Lion C, et al. 2-Hydroxyisoquinoline-1,3(2H,4H)-diones (HIDs), novel inhibitors of HIV integrase with a high barrier to resistance. ACS Chem Biol. 2013;8:1187-94.

24. Sakaki N, Arakawa T, Kozawa H, Yamada Y, Kato H, Kamisawa T. et al. Preliminary study on a novel quadruple eradication therapy with a mucoprotective drug, sofalcone, for Helicobacter pylori infection. J Clin Gastroenterol. 1998;27 Suppl 1: S187-191.

25. Isomoto H, Furusu H, Ohnita K, Wen CY, Inoue K, Kohno S. Sofalcone, a mucoprotective agent, increases the cure rate of Helicobacter pylori infection when combined with rabeprazole, amoxicillin and clarithromycin. World J Gastroenterol. 2005;11:1629-33.

\section{ACKNOWLEDGEMENTS}

We thank Daniela Buccella and Michael Brady for providing 2,8-quinolinedicarboxylic acid, 6-bromo-8-hydroxyquinoline-3 carboxylic acid and 4-oxo-4H-quinolizine-3-carboxylic acid. We thank Thomas M. Moran, Icahn School of Medicine at Mount Sinai, and Luis Martínez-Sobrido, Texas Biomedical Institute, for the kind gift of mouse monoclonal SARS-CoV N antibody 1C7. We thank Hannah Burgess and lan Mohr for providing HCoVOC43 and help in setting up the infection assay. The authors thank Paige Loose for her help with IF work. MP is grateful to T.M. Thor for continuous support.

\section{AUTHOR CONTRIBUTIONS}

$\mathrm{GR}, \mathrm{AZ}$, and BMM conceived, planned, and performed most experiments, and cowrote the manuscript. MV, R.K., AS, and NRL performed and/or provided help with the viral infection assays. SFG and HVG helped with protein purification. HW, TRH, and NZ advised about some biochemical experiments. FB, PC, and DD synthesized Styrylquinoline and NHID compounds. MD directed and coordinated the BSL3 studies. MP conceived, directed, and coordinated the study, oversaw all the results, and co-wrote the manuscript. All authors discussed the results and commented on the manuscript

\section{FUNDING INFORMATION}

This work was funded by grants DA046100, Al122390 and Al120898 from the National Institutes of Health (NIH) to NRL; grants R01Al143639 and R21Al139374 from the NIH to MD, by The G. Harold and Leila Y. Mathers Charitable Foundation to MD, and by grants R01CA76584 and R35GM136250 from the NIH to MP. NZ and MP are Investigators with the Howard Hughes Medical Institute.

\section{COMPETING INTERESTS}

MP is a cofounder of Coho Therapeutics and is on the SAB of, is a consultant for, and has financial interests in Coho Therapeutics, CullGen, Kymera Therapeutics, Santi Therapeutics, and SEED Therapeutics. NZ is a cofounder and SAB member of Coho Therapeutics Inc. and SEED Therapeutics Inc. NZ also serves as a Consultant and SAB member of Kymera Therapeutics. The other authors declare no competing interests. The NYU Grossman School of Medicine has filed an U.S. patent application on work described here and may seek to license the technology.

\section{ETHICS STATEMENT}

Our study did not include human participants or the use of animals, therefore ethical approval was not required.

\section{ADDITIONAL INFORMATION}

Supplementary information The online version contains supplementary material available at https://doi.org/10.1038/s41418-021-00900-1.

Correspondence and requests for materials should be addressed to Meike Dittmann or Michele Pagano.

Reprints and permission information is available at http://www.nature.com/ reprints

Publisher's note Springer Nature remains neutral with regard to jurisdictional claims in published maps and institutional affiliations.

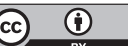

Open Access This article is licensed under a Creative Commons Attribution 4.0 International License, which permits use, sharing, adaptation, distribution and reproduction in any medium or format, as long as you give appropriate credit to the original author(s) and the source, provide a link to the Creative Commons license, and indicate if changes were made. The images or other third party material in this article are included in the article's Creative Commons license, unless indicated otherwise in a credit line to the material. If material is not included in the article's Creative Commons license and your intended use is not permitted by statutory regulation or exceeds the permitted use, you will need to obtain permission directly from the copyright holder. To view a copy of this license, visit http://creativecommons. org/licenses/by/4.0/.

(c) The Author(s) 2021 